\title{
An Intelligent System for Real-Time Condition Monitoring of Tower Cranes
}

\author{
Aaron K. Adik ${ }^{1}$, Wilson Wang2* \\ ${ }^{1}$ Department of Electrical and Computer Engineering, Lakehead University, Thunder Bay, Canada \\ ${ }^{2}$ Department of Mechanical Engineering, Lakehead University, Thunder Bay, Canada \\ Email: ${ }^{\star}$ wilson.wang@Lakeheadu.ca
}

How to cite this paper: Adik, A.K. and Wang, W. (2019) An Intelligent System for Real-Time Condition Monitoring of Tower Cranes. Intelligent Control and Automation, 10, 155-167.

https://doi.org/10.4236/ica.2019.104011

Received: November 1, 2019

Accepted: November 23, 2019

Published: November 26, 2019

Copyright $\odot 2019$ by author(s) and Scientific Research Publishing Inc. This work is licensed under the Creative Commons Attribution International License (CC BY 4.0).

http://creativecommons.org/licenses/by/4.0/

\begin{abstract}
Reliability and safety are major issues in tower crane applications. A new adaptive neurofuzzy system is developed in this work for real-time health condition monitoring of tower cranes, especially for hoist gearboxes. Vibration signals are measured using a wireless smart sensor system. Fault detection is performed gear-by-gear in the gearbox. A new diagnostic classifier is proposed to integrate strengths of several signal processing techniques for fault detection. A hybrid machine learning method is proposed to facilitate implementation and improve training convergence. The effectiveness of the developed monitoring system is verified by experimental tests.
\end{abstract}

\section{Keywords}

Adaptive Neuro-Fuzzy Systems, Machine Learning, Diagnostics, Pattern Classification, Tower Cranes, Smart Sensors

\section{Introduction}

Tower cranes are used extensively at construction sites to lift and move materials. They often rise hundreds of feet into the air, and can reach out just as far. As illustrated in Figure 1, a tower crane consists of several units: 1) the mast is the supporting tower of the crane; 2) slewing unit sits at the top of the mast, which allows the crane to rotate; 3 ) the operator cabin usually sits just above the slewing unit; 4) the jib is the operating arm, along which a trolley runs to move the load; 5) counter jib is the shorter horizontal arm, which carries the hoist unit, control electronics, and the concrete counter weights.

Reliability and safety are the main issues for tower crane applications. Machinery failures in tower cranes could result in catastrophic damage to life and 


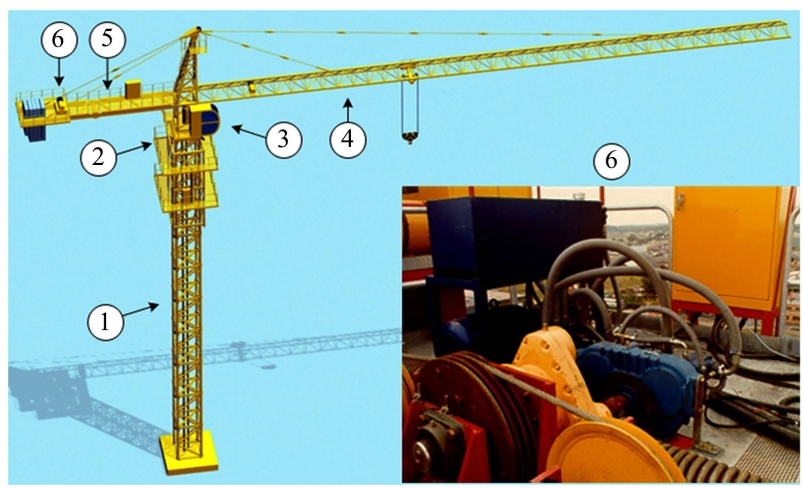

Figure 1. Illustration of a tower crane structure: (1) mast, (2) slewing unit, (3) operator cabin, (4) jib, (5) counter jib, (6) hoist unit.

properties [1]. Several techniques and tools have been proposed in literature for tower crane condition monitoring such as based on the maximum load, maximum moment, maximum lifting height, trolley position on the jib, arm positions to prevent collision, and structure strength [2]. However, unexpected failures always occur in tower crane applications. As a matter of fact, machinery failure of tower cranes has become the top hazard at construction sites [3]. As a result, more strict regulations and clauses have been imposed by the Canadian government [4]; and the manufacturers and contractors have to develop and implement more efficient safety monitoring technologies for tower cranes [5].

This work will focus on the condition monitoring of hoist units in tower cranes. The hoist unit is located in the counter jib as shown in Figure 1. The most dangerous failure in a hoist drive is related gear tooth breakage. The gear materials are subjected to dynamic loading and impacts. If a tooth is broken partly or completely due to fatigue, the following teeth in that gear will be damaged quickly due to extra impacts. The gearbox could become "free rotation", and the lifted load would drop to the ground freely, which could cause serious danger to the people underneath it [6]. New technologies and tools are critically needed for more accurate health condition monitoring of the hoist gearboxes of tower cranes.

Condition monitoring is an act of fault diagnosis by means of appropriate information carriers such as temperature, acoustic signal, or vibration [7]. Vibration-based monitoring is the most commonly used approach because it is ease of measurement and has relatively high signal-to-noise ratio [8], which also will be used in this study.

Fault diagnosis is a process of classifying the vibration features into different categories corresponding to different equipment health conditions. Automatic fault diagnosis can be conducted by either model-based or data-driven techniques [9]. Although model-based approaches are relatively easy to develop and implement, accurate analytical models are difficult to derive especially for complex machinery systems [10]. Data-driven paradigms conduct fault diagnosis by using some intelligent tools such as neural networks, fuzzy logic, or synergetic schemes [11] [12]. The author's research team has also proposed neurofuzzy (NF) 
schemes for machinery fault diagnostics [13] [14], in which the system parameters are trained online/offline. Although these techniques have been applied effectively in many applications [15], these NF schemes could not be used effectively for applications using a standalone computing device with limited RAM capability; in addition they do not have sufficient adaptive capability to accommodate time-varying dynamic effects as in tower crane monitoring applications.

In summary, when a monitoring system is used in real-world applications, unreasonably missed alarms (i.e., the monitor cannot pick up existing faults) and false alarms (i.e., the monitor triggers alarms because of noise instead of real faults) will seriously mitigate its validity. In addition, machinery dynamics may also change suddenly, such as just after repair or regular maintenance. To tackle these aforementioned challenges, the objective of this work is to propose a new adaptive NF (i.e., ANF in short), system for real-time health condition monitoring of hoist gearbox in tower cranes. It is new in the following aspects: 1) A new adaptive NF classifier is proposed for more accurate fault diagnosis in gear systems in order to provide a more reliable real-time condition monitoring tool for tower cranes. 2) A new hybrid training technique is proposed to facilitate training operation and improve training convergence. The effectiveness of the developed classifier is verified by experimental tests.

The remainder of this paper is organized as follows: the proposed intelligent monitoring system is discussed in Section 2. The effectiveness of the proposed techniques is verified experimentally in Section 3.

\section{The Developed Intelligent Monitoring System}

\subsection{Overview}

The developed intelligent monitor consists of several modules as illustrated in Figure 2. The hardware includes smart sensor-based data acquisition (DAQ) system and a computing device (e.g., a small laptop). The software module consists of functions such as signal processing, database management, diagnostic classifier, and training, which will be discussed in this section.

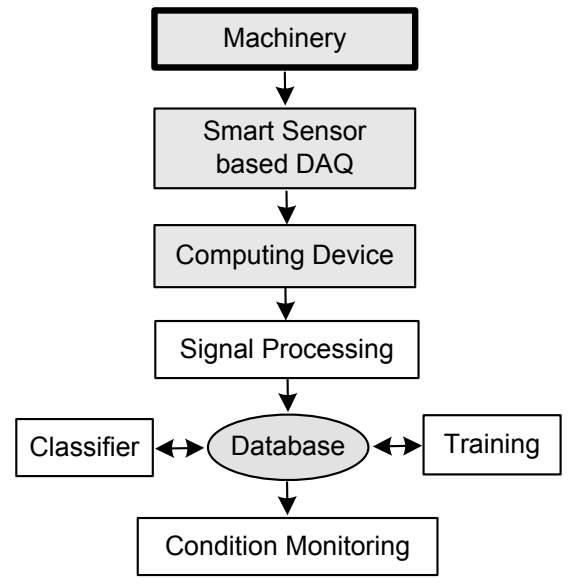

Figure 2. Architecture of the developed intelligent monitor. 


\subsection{Smart Sensor-Based DAQ}

A classical data acquisition system consists of hardware such as sensors, amplifiers, antialiasing filters, and a DAQ board. It is not only expensive but also inconvenient for real-world applications [4]. As the microprocessors become cheaper and faster in processing, it is possible to integrate the related DAQ functions onto chips for smart sensors. The authors' research team has developed many smart sensor-based DAQ systems that can collect different types of signals [16] [17]. In this work, wireless smart vibration and distance sensors are used for tower crane condition monitoring. The vibration sensing unit uses ICP-accelerometers (e.g., PCB 320 series) with a bandwidth of $15,000 \mathrm{~Hz}$, acceleration range of $\pm 140 \mathrm{~g}$, sensitivity of $150 \mathrm{mV} / \mathrm{g}$, and measurement range of $\pm 4900 \mathrm{~m} / \mathrm{s}^{2} \mathrm{pk}$. A distance smart sensor is used to measure gear rotation. Signal conditioning functions (e.g., amplification, rectification, and antialiasing filters) are implemented in IC filter chips so as to reduce size and cost. The wireless communication is based on SWAP protocol with frequency of $915 \mathrm{~Hz}$. A user interface is developed to enable modification of DAQ reconfigurable parameters such as sensor calibration, sampling frequency, filter specifications, monitoring time interval, etc.

\subsection{Adaptive Neurofuzzy Diagnostic Classifier}

A novel ANF diagnostic classifier is developed for gear system monitoring. To improve monitoring accuracy, fault diagnosis is performed gear by gear by the use of time synchronous average filtering [15]. The conditions of each gear are classified into three categories: healthy $\left(C_{1}\right)$, possibly damaged $\left(C_{2}\right)$, and damaged $\left(C_{3}\right)$, respectively. Consider $L$ input variables, $\left\{x_{1}, x_{2}, \cdots, x_{L}\right\}$; three membership functions (MFs), Small, Medium, and Large, are assigned to each input variable. The diagnostic classification, in terms of the diagnostic indicator $y$, can be formulated as

$$
\begin{aligned}
& \mathfrak{R}_{j} \text { : If }\left(x_{1} \text { is } A_{1 j}\right) \text { and }\left(x_{2} \text { is } A_{2 j}\right) \\
& \text { and } \cdots \text { and }\left(x_{L} \text { is } A_{L j}\right) \Rightarrow\left(y \subset C_{j} \text { with } w_{j}\right)
\end{aligned}
$$

where $A_{l j}$ are linguistic MFs of $x_{l}, l=1,2, \cdots, L, j=1,2, \cdots, J$; $J$ is the number of rules; $w_{j}$ is the weight factor (contribution) of the rule $\mathfrak{R}_{j}$ to the diagnostic operation.

The number of rules is associated with the diagnostic reasoning operations of input variables. For example, if the classifier has three inputs $\left\{x_{1}, x_{2}, x_{3}\right\}$ (i.e., $L=3$ ), then diagnostic classification will be performed by the following criteria:

Four rules are associated with healthy condition $\left(C_{1}\right)$ reasoning: If (all three indices are Small), or (two are Small and one is Medium), then (the object is Healthy).

Four rules are associated with damage condition $\left(C_{3}\right)$ formulation: If (all three indices are Large), or (two are Large and one is Medium), then (the object is Damaged). 
Except for these two states, the object is possibly damaged $\left(C_{2}\right)$, and the classification of $C_{2}$ is performed by 9 rules. In all of these cases, different feature association (rule) corresponds to a different weight grade $w_{j}$

Figure 3 schematically shows the network architecture of this diagnostic classifier. Unless specified, all the network links have unity weights. The input nodes in layer 1 transmit the monitoring indices $\left\{x_{1}, x_{2}, \cdots, x_{L}\right\}$ to the next layer. Each node in layer 2 acts as an MF. The nodes in layer 3 perform the fuzzy $T$-norm operations. If a product operator is used, the firing strength of rule $\mathfrak{R}_{j}$ is

$$
\eta_{j}=\prod_{l=1}^{L} A_{l j}\left(x_{l}\right)
$$

where $A_{l j}(\bullet)$ denote MF grades.

Defuzzification is performed in layer 4. If $J_{1}, J_{2}$ and $J_{3}$ are the number of rules associated with the classes $C_{1}, C_{2}$ and $C_{3}$, respectively, the belonging grade to each class is:

$$
\begin{aligned}
& S_{1}=\frac{\sum_{J_{1}} \eta_{j} w_{j}}{\sum_{J_{1}} \eta_{j}} \\
& S_{2}=\frac{\sum_{J_{2}} \eta_{j} w_{j}}{\sum_{J_{2}} \eta_{j}} \\
& S_{3}=\frac{\sum_{J_{3}} \eta_{j} w_{j}}{\sum_{J_{3}} \eta_{j}}
\end{aligned}
$$

The diagnostic indicator will be

$$
y=S_{1} \vee S_{2} \vee S_{3} .
$$

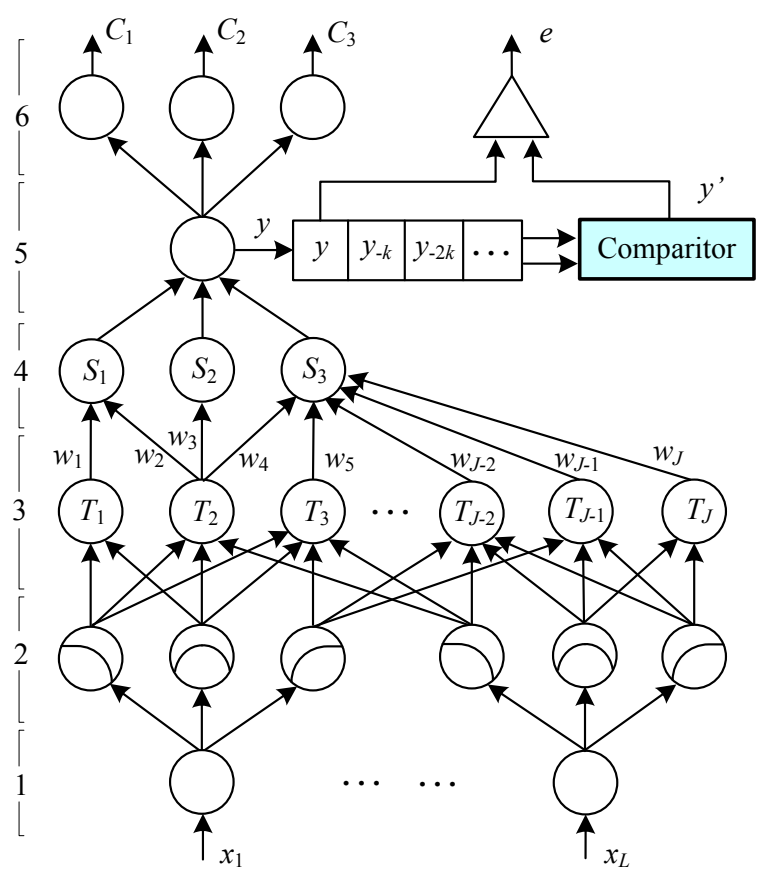

Figure 3. The network architecture of the diagnostic classifier. 


\subsection{Diagnostic Decision-Making}

In machinery health condition monitoring, if the diagnostic indicator $y \leq \varepsilon_{1}$, the object is considered healthy; $\varepsilon_{1}$ is a threshold ( $\varepsilon_{1}=0.50$ in this case). If $\varepsilon_{1}<y \leq \varepsilon_{2}$, the monitored object is possibly damaged; $\varepsilon_{2}$ is another threshold ( $\varepsilon_{2}=0.70$ in this case). Otherwise, if $y>\varepsilon_{2}$, the object is damaged. An appropriate alarm should be given to the user for the occurrence of the defect.

When the indicator $y$ values are formulated, the corresponding historical indicator $y^{\prime}$ in the database will be used to verify the diagnostic results so as to improve diagnostic reliability. In operation, the objective is to minimize the match error, $e=\left|y-y^{\prime}\right|$, between the current value of the diagnostic indicator $y$ and its comparator value $y^{\prime}$. If $y$ and $y^{\prime}$ belong to different classes, for example $C_{1}$ (healthy) and $C_{2}$ (possibly damaged), there are three possible reasons: 1) the defect recognized by the ANF classifier is caused by noise instead of real component damage; 2) the historical comparator data sets are not accurate; and 3) the convergence of the ANF classifier deteriorates due to local minima. These problems can be solved by the training process. For the first reason, the error-making rules will be punished to reduce their weight factors $w_{j}$ to the diagnostic classification. If the database is not accurate, it will be updated using more accurate data sets for future monitoring and training application. If the errors are caused by possible trapping of location minima, the rule boundary properties in the decision space will be modified by appropriate training operations.

\subsection{Hybrid System Training}

Once the ANF classifier is set up, it should be properly trained to improve its performance. In this case, a hybrid training strategy is suggested to update system parameters. The linear parameters will be trained by the use of the classical least squires estimator (LSE).

Many algorithms have been proposed in literature for nonlinear parameter optimization, each having its merits and limitations. The Levenberg-Marquardt (L-M) algorithm possesses quadratic convergence close to a minimum [18]. Its convergence is still reasonably well even if the initial estimates are relatively poor. This characteristic is important in the developed real-time monitoring applications. In addition, the L-M algorithm has been proven globally convergent in many applications by properly choosing the step factors. In this monitoring application, the system uses a less powerful standalone computing device (e.g., a small laptop computer), which has limited RAM space. In using the L-M algorithm, when the number of intermediate variables becomes large in operation, the computation of the inverse of a large size Hessian matrix will take a very long time. The excessive computing load can dramatically slow down or even block the computing operations. To solve this problem, a novel recessive L-M training method is suggested in this work to facilitate its implementation and improve its convergence.

The objective or error function with respect to adjustable parameters $\boldsymbol{\theta}_{k}$ at the current time instant $k$ is defined as 


$$
E\left(\boldsymbol{\theta}_{k}\right)=\frac{1}{2} \sum_{k=1}^{K}\left[y_{k}\left(\boldsymbol{\theta}_{k}\right)-y_{d}\right]^{2}=\frac{1}{2} \sum_{k=1}^{K} r_{k}^{2}\left(\boldsymbol{\theta}_{k}\right)=\boldsymbol{r}_{k}^{\mathrm{T}}\left(\boldsymbol{\theta}_{k}\right) \boldsymbol{r}_{k}\left(\boldsymbol{\theta}_{k}\right)
$$

where $y_{k}\left(\boldsymbol{\theta}_{k}\right)$ is the $k$ th output, $k=1,2, \cdots, K ; y_{d}$ is the desired output. $\boldsymbol{r}_{k}\left(\boldsymbol{\theta}_{k}\right)$ is the error vector that can be either linear or nonlinear.

To simplify expression, the variable $\boldsymbol{\theta}_{k}$ is dropped in the related terms in these manipulations. By taking the Tayler series expansion and neglecting higher order terms,

$$
\begin{aligned}
\boldsymbol{\theta}_{k+1} & \approx \boldsymbol{\theta}_{k}+\lambda_{k}\left(\boldsymbol{J}_{k}^{\mathrm{T}} \boldsymbol{J}_{k}+\eta_{k} \boldsymbol{I}\right)^{-1} \boldsymbol{J}_{k}^{\mathrm{T}} \boldsymbol{r}_{k}=\boldsymbol{\theta}_{k}+\lambda_{k} \boldsymbol{H}_{k}^{-1} \boldsymbol{J}_{k}^{\mathrm{T}} \boldsymbol{r}_{k} \\
& =\boldsymbol{\theta}_{k}+\left(1-\alpha_{k}\right) \boldsymbol{H}_{k}^{-1} \boldsymbol{J}_{k}^{\mathrm{T}} \boldsymbol{r}_{k}
\end{aligned}
$$

where $\boldsymbol{J}_{k} \in \mathbb{R}^{Z \times Z}$ is the Jacobin matrix; $Z$ is dimension of $\boldsymbol{\theta}_{k}$ (or the number of parameters to be trained); $\boldsymbol{H}_{k} \in \mathrm{R}^{Z \times Z}$ is the Hessian matrix; $\boldsymbol{I} \in \mathbb{R}^{Z \times Z}$ is an identity matrix; $\lambda_{k}=1-\alpha_{k} ; \alpha_{k}$ is the forgetting factor; $\eta_{k}$ is the learning that can be adapted by a line search method.

The Hessian matrix can be expressed as

$$
\boldsymbol{H}_{k}=\alpha_{k} \boldsymbol{H}_{k-1}+\left(1-\alpha_{k}\right)\left(\boldsymbol{J}_{k}^{\mathrm{T}} \boldsymbol{J}_{k}+\eta_{k} \boldsymbol{I}\right) .
$$

In implementation, instead of computing the $Z \times Z$ matrix $\eta_{t} \boldsymbol{I}$ at each time step, $\eta_{t}$ is added to one of the diagonal elements of $\boldsymbol{J}_{k}^{\mathrm{T}} \boldsymbol{J}_{k}$ at each time instant

$$
\boldsymbol{H}_{k}=\alpha_{k} \boldsymbol{H}_{k-1}+\left(1-\alpha_{k}\right)\left(\boldsymbol{J}_{k}^{\mathrm{T}} \boldsymbol{J}_{k}+Z \eta_{k} \boldsymbol{\Lambda}\right)
$$

where $\Lambda \in \mathrm{R}^{Z \times Z}$ has only one nonzero element located at $t\{\bmod (Z)+1\}$ diagonal position, or

$$
\Lambda_{i i} \in \begin{cases}1, & \text { if } i=k\{\bmod (Z)+1\}, \text { and } k>Z \\ 0, & \text { otherwise }\end{cases}
$$

Correspondingly, Equation (8) can be rewritten as

$$
\boldsymbol{H}_{k}=\alpha_{k} \boldsymbol{H}_{k-1}+\left(1-\alpha_{k}\right)\left(\boldsymbol{U} \boldsymbol{V}^{-1} \boldsymbol{U}^{\mathrm{T}}\right)
$$

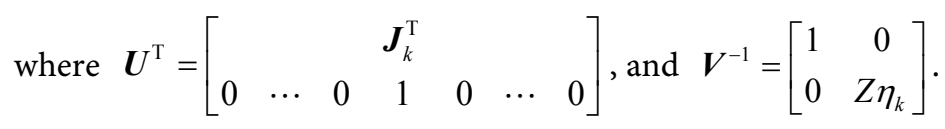

The computation of $\boldsymbol{H}_{k}^{-1}$ in Equation (6) is very time consuming, and it not suitable for real-time applications. Here $\boldsymbol{H}_{k}^{-1}$ is computed by using following approach:

$$
(\boldsymbol{A}+\boldsymbol{B C D})^{-1}=\boldsymbol{A}^{-1}-\boldsymbol{A}^{-1} \boldsymbol{B}\left(\boldsymbol{C}^{-1}+\boldsymbol{D} \boldsymbol{A}^{-1} \boldsymbol{B}\right)^{-1} \boldsymbol{D} \boldsymbol{A}^{-1}
$$

where $\boldsymbol{A}, \boldsymbol{B}, \boldsymbol{C}$ and $\boldsymbol{D}$ are matrices such that $\boldsymbol{A}$ and $\left(\boldsymbol{C}^{-1}+\boldsymbol{D} \boldsymbol{A}^{-1} \boldsymbol{B}\right)$ are nonsingular matrices.

Based on Equation (11), Equation (6) can be rewritten as

$$
\begin{aligned}
\boldsymbol{\theta}_{k+1}= & \boldsymbol{\theta}_{k}+\left(1-\alpha_{k}\right) \boldsymbol{H}_{k}^{-1} \boldsymbol{J}_{k}^{\mathrm{T}} \boldsymbol{r}_{k} \\
= & \boldsymbol{\theta}_{k}+\left(1-\alpha_{k}\right) \times\left\{\left(\alpha_{k} \boldsymbol{H}_{k-1}\right)^{-1}-\left(\alpha_{k} \boldsymbol{H}_{k-1}\right)^{-1}\left(1-\alpha_{k}\right) \boldsymbol{U}\right. \\
& \left.\times\left[\boldsymbol{V}+\boldsymbol{U}^{\mathrm{T}}\left(\alpha_{k} \boldsymbol{H}_{k-1}\right)^{-1}\left(1-\alpha_{k}\right) \boldsymbol{U}\right]^{-1} \boldsymbol{U}^{\mathrm{T}}\left(\alpha_{k} \boldsymbol{H}_{k-1}\right)^{-1}\right\} \boldsymbol{J}_{k}^{\mathrm{T}} \boldsymbol{r}_{k}
\end{aligned}
$$


Let $\boldsymbol{A}=\alpha_{k} \boldsymbol{H}_{k-1}, \quad \boldsymbol{B}=\left(1-\alpha_{k}\right) \boldsymbol{U}, \boldsymbol{C}=\boldsymbol{V}^{-1}$, and $\boldsymbol{D}=\boldsymbol{U}^{\mathrm{T}}$. From Equation (12), the recursive L-M algorithm can then be represented by

$$
\boldsymbol{\theta}_{k+1}=\boldsymbol{\theta}_{k}+\boldsymbol{\Phi}_{k} \boldsymbol{J}_{k} \boldsymbol{r}_{k}
$$

where $\boldsymbol{\Phi}_{k}=\frac{1}{\alpha_{k}}\left[\boldsymbol{\Phi}_{k-1}-\boldsymbol{\Phi}_{k-1} \boldsymbol{U} \boldsymbol{S}^{-1} \boldsymbol{U}^{\mathrm{T}} \boldsymbol{\Phi}_{k-1}\right]$,

$$
\boldsymbol{S}=\alpha_{k} \boldsymbol{V}+\boldsymbol{U}^{\mathrm{T}} \boldsymbol{\Phi}_{k-1} \boldsymbol{U}
$$

$S$ is a matrix with dimension $2 \times 2$; its inverse computation is simple and can be implemented for real-time monitoring applications. $\boldsymbol{\theta}_{0}=\mathbf{0} ; \boldsymbol{\Phi}_{t}$ is a covariance matrix with initial condition $\Phi_{0}=\rho \boldsymbol{I}$, where $\rho \in\left[10^{2}, 10^{6}\right]$, $\eta_{k} \in[0.001,10]$, and $\alpha_{k} \in[0.95,1]$.

In hybrid training, as each data sample is inputted to the ANF classifier, the linear consequent parameters $w_{j}$ are updated by using the LSE. The nonlinear classifier MF parameters are trained by the use of the recursive L-M method. On the other hand, adaptive training is preferred in real-time applications because: 1 ) it is necessary for time-varying systems; 2) it possesses randomness that may help to escape from a local minimum; and 3) it is useful when the number of training data is large.

\section{Performance Evaluation}

The effectiveness of the proposed ANF classifier techniques will be verified in this section by the use of experimental tests.

\subsection{Experimental Setup}

The experimental setup used in this study is schematically shown in Figure 4 . The system is driven by a 3-HP induction motor. A pair of gears are tested in this case. The loading is provided by heavy-duty magnetic breaks connected to the gearbox. The motor rotation is controlled by a speed controller, which allows tested gearbox operation in the range of 20 to $3600 \mathrm{rpm}$. The vibration is measured using two smart vibration sensors (accelerometers) mounted on the gearbox housing. A displacement sensor is used to provide gear rotating information, which is used as a reference for time synchronous average filtering. The collected signals are wirelessly fed to a receiver that is connected to a computer through a USB port.

\subsection{Monitoring Indices}

Gear fault consists of localized damage and distributed defects (e.g., wear and pitting). This work focuses on localized gear fault diagnosis because a localized fault can not only generate transmission errors but also may cause sudden failures [15] [19]. For example, a tiny crack may occur at a gear tooth due to dynamic loading, stress concentration, or impacts. As the gear system continues in operation, the crack propagates continuously. Unfortunately, by the time that the crack is visible, the gear may be more than $90 \%$ used up. When a tooth is 


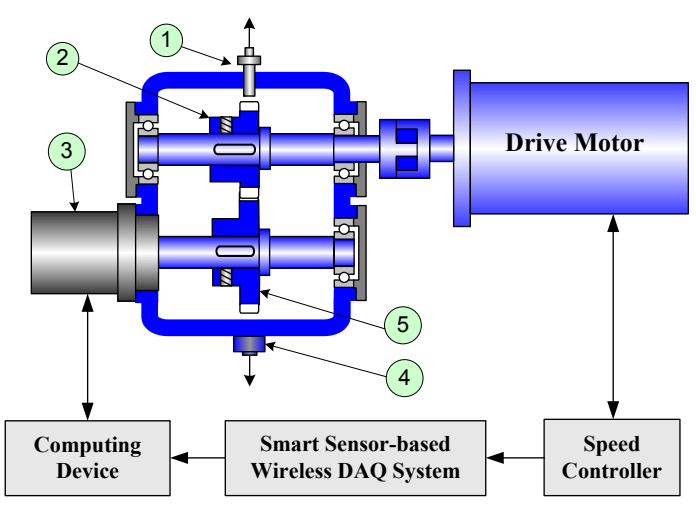

Figure 4. Experimental setup: (1) the smart displacement sensor, (2) the driving gear, (3) the load drive, (4) the smart vibration sensor, (5) the driven gear.

broken out, the following teeth will be damaged quickly due to extra impacts, which could induce severe accidents in tower crane applications.

One of the initiatives of this work is to perform fault diagnosis of the gearbox gear by gear. Because the measured vibration is an overall signal generated from various rotary sources, the first step is to differentiate the signal specific to each gear by using the time synchronous average filter [15]. In this filtering process, all of the signals that are non-synchronous to the rotation of the gear of interest (e.g., those from bearings and shafts) are filtered out. As a result, each gear signal is computed and represented in one full revolution, called the signal average, which will be used for advanced analysis.

Several techniques have been proposed in the literature for gear fault detection; however, each has its own advantages and limitations, and is efficient for specific applications only [13] [14]. As a result, the more features are applied in diagnostic classification, the more possible it is to achieve conflicting diagnostic results. The selected features should be robust, that is, sensitive to component defects but insensitive to noise (i.e., the signal not carrying health information). By investigation, three features are selected in this work from different information domains: energy, amplitude, and phase:

1) wavelet energy function, using the overall residual signal that is obtained by bandstop filtering out the gear mesh frequency $f_{R} N$ and its harmonics, where $f_{R}$ is the rotation frequency (in $\mathrm{Hz}$ ) of the gear of interest and $N$ is the number of teeth of the gear;

2) beta kurtosis, using the overall residual signal;

3) phase demodulation using the signal average. Details of these techniques can be found from [15].

\subsection{Performance Evaluation by Experiment}

To verify the effectiveness of the developed intelligent monitor and the related techniques, systematic tests have been conducted corresponding to different machinery conditions. Three gear cases are tested in this case, as illustrated in Figure 5: (a) healthy gears, (b) cracked gears, and (c) scored gears. 


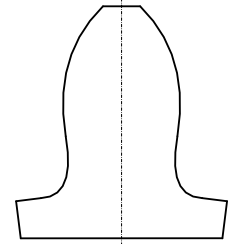

(a)

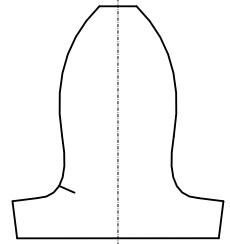

(b)

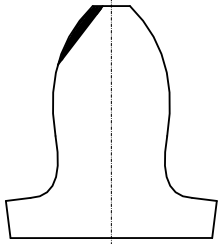

(c)

Figure 5. Tested gears: (a) Healthy gears; (b) Cracked gears; (c) Scored gear.

To make a comparison, two other related diagnostic classifiers are considered:

1) Classifier-1: A general ANFIS classifier, but using the proposed hybrid training technique with the LSE and the recursive L-M for training. It is to compare the proposed ANF classification scheme.

2) Classifier-2: It has the same network architecture with the ANF classifier, but the training is using the LSE and the classical L-M algorithm. It is to compare training efficiency of the proposed hybrid training based on the recursive L-M algorithm.

A series of tests have been conducted corresponding to different gear conditions. Firstly, both gears are healthy. The tests are undertaken with different load levels and speed. 225 data sets are collected for system training (120 pairs) and testing (105 pairs). Then the driven gear with a simulated crack is installed and tested. 300 data sets are collected; 200 pairs are used for training and 100 pairs for testing. In scored gear testing, similarly, 300 sets of data are collected; 200 pairs are used for training and 100 pairs for testing. Test results are summarized in Table 1, in which FA stands for False Alarms, and MA stands for Missed Alarms.

It is seen the developed ANF classifier provides best performance in this test, which has recorded 3 missed alarms and 2 false alarms during the testing periods. The missed alarms are mainly induced when the motor rotates at a very low speed, and the resulting signature modulation due to a small tooth crack is very weak. The false alarms are caused by the dramatic speed and load variations in testing. ANF classifier performs better than Classifier 1 that generates 5 missed alarms and 7 false alarms. It can verify the better classification efficiency of the proposed ANF diagnostic classification scheme. The developed ANF classifier also outperforms Classifier-2 that has recorded 4 missed alarms and 4 false alarms during the testing periods. It can verify the effective convergence of the proposed the recursive L-M algorithm.

\subsection{Monitoring of the Hoist Gearbox}

The purpose of the developed ANF classifier is for hoist gearbox condition monitoring and diagnostics. Figure 6 schematically shows the structure of a hoist drive in a tower crane. The gearbox consists of two pairs of gears. The monitoring is taken gear-by-gear. Vibration signals are collected using the smart vibration sensors. The gear rotation signal is measured by a wireless encoder, which 
provides a reference for time synchronous average filtering.

Figure 7 shows a demo of an interface for this application example. The processing results are from the developed ANF monitoring system. It shows the current diagnostic indicator values of each gear, as well as the comparator values. To facilitate monitoring, the green color is used to specify the "healthy state", whereas yellow and red colors indicate "possibly damaged" and "damaged" conditions, respectively.

Table 1. Summary of the testing results using different classifiers (FA: False Alarms, MA: Missed Alarms).

\begin{tabular}{|c|c|c|c|c|c|c|c|}
\hline \multirow{2}{*}{$\begin{array}{c}\text { Diagnostic } \\
\text { Classifier }\end{array}$} & \multicolumn{2}{|c|}{$\begin{array}{l}\text { Healthy } \\
\text { Gears }\end{array}$} & \multicolumn{2}{|c|}{$\begin{array}{l}\text { Cracked } \\
\text { Gears }\end{array}$} & \multicolumn{2}{|c|}{$\begin{array}{l}\text { Chipped } \\
\text { Gears }\end{array}$} & \multirow[t]{2}{*}{$\begin{array}{c}\text { Overall } \\
\text { Accuracy } \\
\%\end{array}$} \\
\hline & MA & FA & MA & FA & MA & FA & \\
\hline Classifier-1 & 1 & 3 & 2 & 2 & 2 & 2 & 88.4 \\
\hline Classifier-2 & 0 & 2 & 3 & 1 & 1 & 1 & 94.2 \\
\hline $\begin{array}{c}\text { ANF } \\
\text { Classifier }\end{array}$ & 0 & 1 & 2 & 1 & 1 & 1 & 98.8 \\
\hline
\end{tabular}

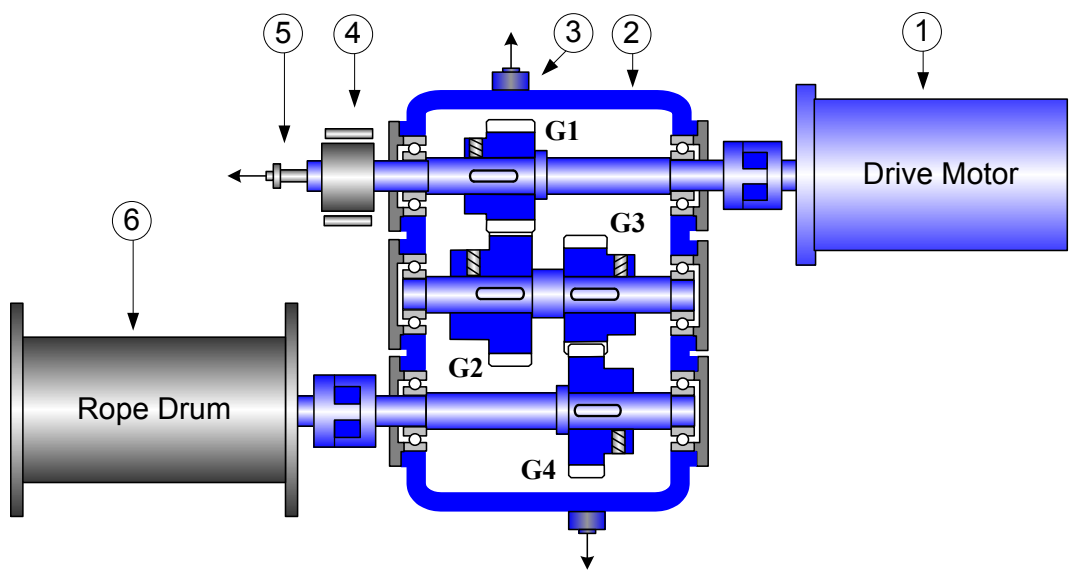

Figure 6. A hoist drive unit: (1) drive motor, (2) gearbox, (3) smart vibration sensors, (4) brake clutch, (5) encoder, (6) rope drum.

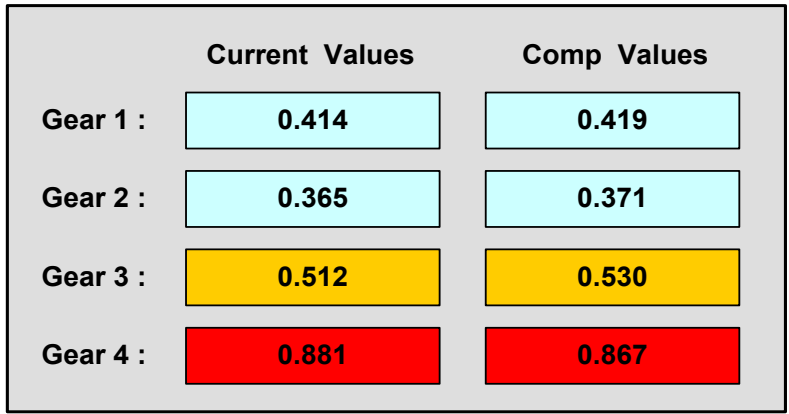

Figure 7. An interface example of the developed intelligent motor. 


\section{Conclusion}

A new ANF monitoring system is developed in this work for health condition monitoring of tower cranes, especially for hoist gearboxes. Vibration signals are measured using wireless smart sensors. Fault detection is performed gear-by-gear. A new ANF diagnostic classifier is developed to integrate strengths of several signal processing techniques for more accurate fault diagnosis. A novel hybrid training method based on a recursive L-M technique is proposed to improve processing efficiency by reducing the matrix size, and enhance the training convergence. The effectiveness of the proposed diagnostic techniques has been verified by experimental tests corresponding to different gear and operating conditions. Test results have demonstrated that the developed ANF monitoring system can provide more reliable fault diagnostics of the gear systems. It has also been implemented for real tower crane hoist drive system monitoring applications.

\section{Acknowledgements}

This work was supported in part by Natural Sciences and Engineering Research Council of Canada (NSERC), Bare Point Water Treatment Plant, and eMech Systems Inc.

\section{Conflicts of Interest}

The authors declare no conflicts of interest regarding the publication of this paper.

\section{References}

[1] Shaoiro, L.K. and Shapiro, J.P. (2010) Cranes and Derricks. 4th Edition, McGraw-Hill, New York.

[2] Zhao, C., Zhang, J., Zhong, X., Chen, S. and Liu, X. (2012) Analysis of Tower Crane Monitoring and Life Prediction. Journal of Adaptive \& Dynamic Computing, 2, 12-16. https://doi.org/10.5813/www.ieit-web.org/IJADC/2012.2.3

[3] Raviv, G., Shapira, A. and Fishbain, B. (2017) AHP-Based Analysis of the Risk Potential of Safety Incidents: Case Study of Cranes in the Construction Industry. Safety Science, 91, 298-309. https://doi.org/10.1016/j.ssci.2016.08.027

[4] Ni, Y., Li, B., Lam, K., Zhu, D., Wang, Y., Lynch, J. and Law, K. (2011) In-Construction Vibration Monitoring of a Super-Tall Structure Using a Long-Range Wireless Sensing System. Smart Structures and Systems, 7, 83-102. https://doi.org/10.12989/sss.2011.7.2.083

[5] Ministry of Labor (2011) Safety of Tower Cranes in Construction.

[6] Qu, Y., He, D., Yoon, J., Hecke, B., Bechhoefer, E. and Zhu, J. (2017) Gearbox Tooth Cut Fault Diagnostics Using Acoustic Emission and Vibration Sensors-A Comparative Study. Archives of Computational Methods in Engineering, 24, 543-556.

[7] Randall, R. (2011) Vibration-Based Condition Monitoring. Wiley, New York. https://doi.org/10.1002/9780470977668

[8] Hong, L. and Dhupia, J. (2014) A Time Domain Approach to Diagnose Gearbox Fault Based on Measured Vibration Signals. Journal of Sound and Vibration, 333, 
2164-2180. https://doi.org/10.1016/j.jsv.2013.11.033

[9] Jardine A. and Banjevic, D. (2008) Condition Monitoring. In: Encyclopedia of Quantitative Risk Assessment, John Wiley \& Sons, Hoboken, 341-346. https://doi.org/10.1002/9780470061596.risk0502

[10] Vachtsevanos, G., Lewis, F., Roemer, M., Hess, A. and Wu, B. (2006) Intelligent Fault Diagnosis and Prognosis for Engineering Systems. Wile, Hoboken. https://doi.org/10.1002/9780470117842

[11] Jing, L., Zhao, M., Li, P. and Xu, X. (2017) A Convolutional Neural Network Based Feature Learning and Fault Diagnosis Method for the Condition Monitoring of Gearbox. Measurement, 111, 1-10. https://doi.org/10.1016/j.measurement.2017.07.017

[12] Cerrada, M., Sánchez, R., Pacheco, F., Cabrera, D., Zurita, G. and Li, C. (2016) Hierarchical Feature Selection Based on Relative Dependency for Gear Fault Diagnosis. Applied Intelligence, 44, 687-703. https://doi.org/10.1007/s10489-015-0725-3

[13] Wang W. and Kanneg, D. (2009) An Integrated Classifier for Condition Monitoring in Transmission Systems. Mechanical Systems and Signal Processing, 23, 1298-1312. https://doi.org/10.1016/j.ymssp.2008.10.006

[14] Modi V. and Wang, W. (2018) A Constrained Evolving Classifier for Gearbox Health Monitoring. Journal of Mechanical and Mechatronics Engineering, 18, 1-7.

[15] Wang, W. (2008) An Enhanced Diagnostic System for Gear System Monitoring. IEEE Transactions on Systems, Man and Cybernetics, Part B: Cybernetics, 38, 102-112. https://doi.org/10.1109/TSMCB.2007.908864

[16] Wang, W. and Jianu, O. (2010) A Smart Monitor for Machinery Fault Detection. IEEE/ ASME Transactions on Mechatronics, 15, 70-78. https://doi.org/10.1109/TMECH.2009.2016956

[17] Luong, P. and Wang, W. (2019) Smart Sensor-Based Synergistic Analysis for Rotor Bar Fault Detection of Induction Motors. IEEE/ ASME Transactions on Mechatronics. (In Press)

[18] Wang, W. and Vrbanek, J. (2008) An Evolving Fuzzy Predictor for Industrial Applications. IEEE Transactions on Fuzzy Systems, 16, 1439-1449. https://doi.org/10.1109/TFUZZ.2008.925918

[19] Osman, S. and Wang, W. (2018) A Leakage-Free Resonance Sparse Decomposition Technique for Bearing Fault Detection in Gearboxes. Measurement Science and Technology, 29, Article ID: 035004. https://doi.org/10.1088/1361-6501/aa9d5a 